\title{
Aggregated Silver Sols as SERS Substrates
}

\author{
S. Kruszewski* And M. Cyrankiewicz \\ Medical Physics Division, Biophysics Department, Collegium Medicum of Nicolaus Copernicus University \\ Jagiellońska 13, 85-067 Bydgoszcz, Poland

\begin{abstract}
The unique plasmonic properties of the noble metal colloidal nanoparticles make them promising enhancement substrates for surface-enhanced Raman scattering. Obtaining of systems that provide ever-increasing enhancement of the Raman scattered light is a big challenge. Silver colloids studied here are prepared by Lee-Meisel's method. Rhodamine $6 \mathrm{G}$ and rhodamine $\mathrm{B}$ are used as probe adsorbates. The "raw" colloids obtained in this way exhibit a characteristic extinction band proving surface plasmons excitation, but do not enhance the Raman signal. Theoretical calculations indicate that the extremely large electromagnetic field is induced in the junctions between metallic nanostructures so some degree of their aggregation is necessary to achieve a sufficient gain value. The addition of aggregating agents $(\mathrm{KCl}$ or $\mathrm{HCl}$ ) leads to significant changes in the extinction spectrum and to significant increase in the intensity of surface-enhanced Raman scattering. The experiments show that chloride can not only promote the aggregation process but also effectively affect chemical mechanisms contributing to surface-enhanced Raman scattering.
\end{abstract}

PACS: 33.20.Fb, 33.20.Kf, 33.50.-j

\section{Introduction}

Silver and gold nanoparticles are the systems which are able to enhance the intensity of the Raman scattered light. The first experimental and theoretical reports on surface-enhanced Raman scattering (SERS) [1-3] appeared in the 70's of 20th century. The earlier reports focused on SERS on silver electrodes with modified roughened surfaces, but since 1979 when enhancement of the Raman signal on silver and gold sols was observed the first time [4] the colloidal metal particles have become the most commonly used substrates.

Silver and gold nanoparticles, because of its unique properties (resonance excitation of surface plasmons and associated with this phenomenon resonance light absorption, resonance light scattering and ability of enhancement of intensity of the Raman scattered light) find wide application in medical diagnosis and therapy. The most spectacular applications of nanoparticles and SERS phenomenon are uses of antibodies functionalized gold nanoparticles in detecting and destroying of cancer cells $[5-8]$.

The enhancement of intensity Raman scattered light is mainly a result of electric field enhancement of both incident and scattered lights on the metal nanoparticles, e.g. on nanostructures forming the rough surface $[9,10]$ or on nanoparticles dispersed in solution. This enhancement called as electromagnetic (EM) enhancement is related to the resonance excitation of surface plasmons localized in

* corresponding author; e-mail: skrusz@cm.umk.pl metal nanoparticles. Plasmons excited in metal nanoparticles have the characteristic frequencies depending on sizes, shapes and dielectric functions of nanoparticles and surrounding medium [11, 12]. Conditions for occurring of large EM enhancement are fulfilled for silver, gold and copper, only. If metallic nanoparticles are placed in the field of light and frequency of this light is equal or close to frequency of plasmonic oscillations on the surface of these nanoparticles, the resonance enhancement of electric field of light occurs [13]. If molecules are placed in sites, where electric field of light is enhanced, the intensity of the Raman scattered light is also enhanced, locally even more than $10^{6}$ fold. An additional enhancement is provided by chemical mechanism. It is associated with the increase in polarizability of molecules adsorbed on metal - for adsorbed molecules exciting light can cause charge transfer (CT) transition from (or to) the Fermi level of metal to (or from) levels of adsorbed molecules and thus condition for resonance Raman scattering can be fulfilled. The total enhancement provided by chemical mechanism usually does not exceed $10^{2}$.

The optimal conditions for SERS enhancement are fulfilled only in the small part of the surface and surroundings of nanoparticles. As a result, for colloids containing isolated nanoparticles, total enhancement does not exceed $10^{6}$ and usually is not greater than two orders of magnitude. Enhancement properties of colloid essentially change by the aggregation of nanoparticles. Then, the large enhancement of electric field occurs in "junctions" between nanoparticles. Value of this enhancement depends on width of gaps (smaller width - larger enhancement) and on curvature of nanoparticles (larger 
curvature - larger enhancement). If molecules of analyte are placed in the internal "junctions" in aggregates illuminated by the excitation light then the intensity of the Raman scattered light can be enhanced even $10^{15}$ fold (as the result of both mechanisms, EM and CT). It means that the presence of few molecules placed in the narrow slits between nanoparticles is sufficient for recording of SERS spectrum. Phenomenon of such giant enhancement was first time observed in 1996 by two independent research groups [14, 15] and was called single molecules SERS (SM SERS), and sites into which molecules should be placed for occurring such big enhancement were called "hot spots". Obtaining of colloid containing aggregated nanoparticles with large number of "hot spots" is a big challenge. One of the main aims of this work is the investigation of the influence of colloid aggregation process on the intensity of SERS spectra of rhodamine $6 \mathrm{G}$ and rhodamine B adsorbed on silver nanoparticles. Observed increase in SERS signal may prove forming of "junctions" into which conditions for SM SERS are fulfilled.

In the next part of this paper results of theoretical calculations of the electric field distribution on the surface and in vicinity of spherical silver nanoobjects, both isolated and assembled into dimer, are shown. Values of the electric field enhancement and SERS enhancement in the gaps between adjacent nanospheres are calculated. Theoretical extinction spectra of silver nanospheres for the different sizes are presented. In experimental parts of this work the silver sol aggregation processes are studied. Influence of the nanoparticles aggregation on SERS signal and extinction spectra are presented and discussed.

\section{Electromagnetic field around the nanoparticles}

The first general description of elastic scattering of plane electromagnetic wave on isolated spherical objects was made in the early twentieth century by Mie [16]. In subsequent decades the Mie (sometimes called LorenzMie) method has been intensively developed. The original theory was also extended to describe the scattering of light on systems of particles freely distributed in space and differing in size and material properties. One of such generalization called generalized multiparticle Mie-solution (GMM) proposed by $\mathrm{Xu}[17]$ is used in this work. This method is still the subject of intense work whose aim is to improve the efficiency and stability of numerical algorithm. It is noteworthy that the results obtained using GMM exhibit excellent agreement with experiments [18].

In this chapter some aspects of the near field problem are illustrated by the results of authors' calculations. The energy flow (the Poynting vector) and electric field enhancement around isolated metallic nanospheres are calculated from original Mie theory. However, the distribution of near field around an ensemble of nanoparticles as well as the enhancement factor for SERS of molecules placed between them are obtained using GMM approach.

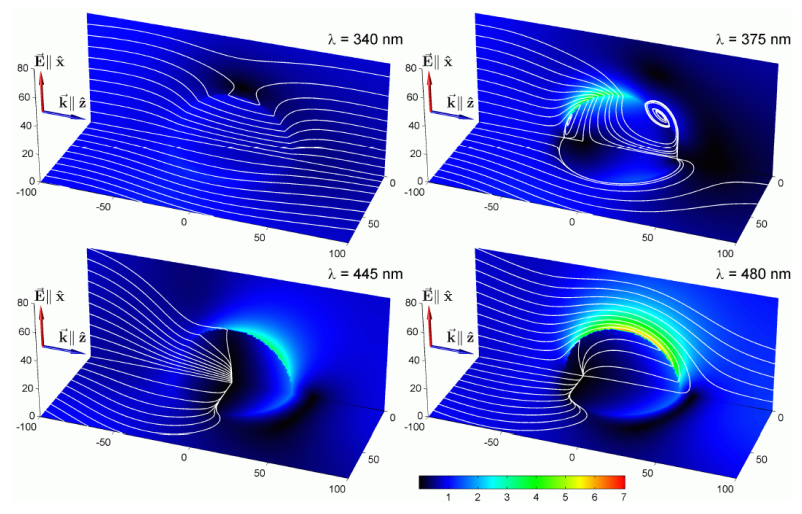

Fig. 1. Time-averaged EM energy density distribution around the spherical silver nanoparticles with radius $a=39 \mathrm{~nm}$ illuminated by light of various wavelengths. The unit on the scale of colorbar represents the energy density of incident radiation. White lines represent the direction of energy flow (direction of the Poynting vector).

In Fig. 1 one can see the complex pattern of energy flow in the near-field region surrounding a silver nanoparticle. The graphic also shows how it changes with different frequency of incident light. The illustration shows a few interesting phenomena occurring in the vicinity of plasmon resonance, in particular, the existence of singularities and the formation of optical vortices. These phenomena are discussed in detail in [19]. From point of view of this paper the most interesting is the fact that close to the resonance condition lines of energy flux can enter into the nanoparticle not only from the front (with respect to a power source) but also from the "shadow" side. As a result the nanoparticle can absorb much more radiation than that given by its geometrical cross-sections.

Figure 2 shows the near field distributions in the vicinity of spherical nanoparticle with a radius of $39 \mathrm{~nm}$ (nanoparticles with this size are the subject of experimental studies carried out in this work) placed in water. The wavelength of incident radiation is $445 \mathrm{~nm}$. One can see that the electric field mainly concentrates around the poles of nanoparticle (with respect to the direction of electric field of incident EM wave). The enhancement of electric field predicted by the Mie theory can reach up to 7 -fold in these regions.

In the case of small spherical particles (with diameters significantly smaller than the wavelength) oscillations induced on their surface can be treated as a dipole so the largest electric field appears at the poles and its spatial distribution is almost symmetric with respect to the direction of propagation of light. The situation changes in the case of larger particles. Due to a significant contribution of higher multipoles the field distribution is no longer symmetric and areas of major enhancement appear outside the poles (see Fig. 3).

The much stronger EM field (in comparison with isolated nanoparticles presented in Fig. 2 and Fig. 3) is induced inside an aggregate of nanoparticles, especially in narrow gaps between them, in so-called "hot spots". 


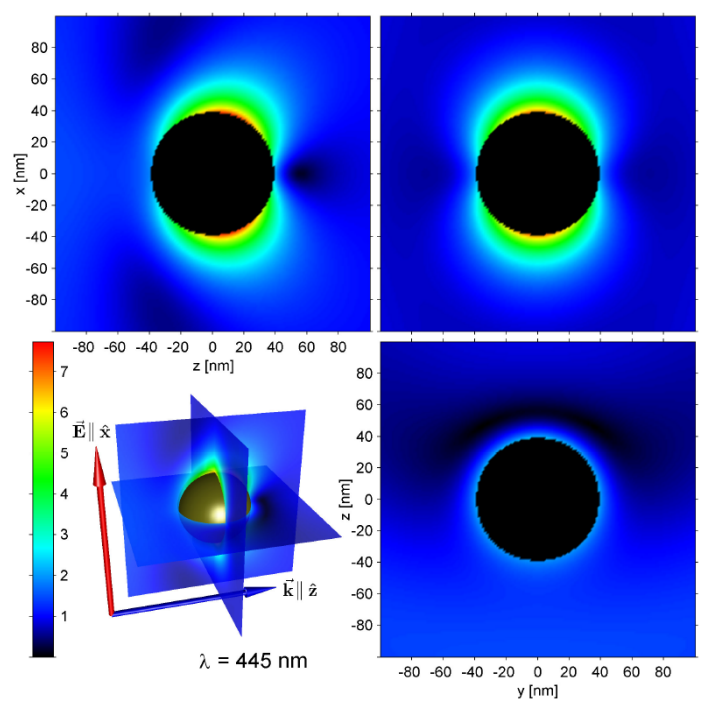

Fig. 2. Local electric field distribution around a single silver sphere with radius $a=39 \mathrm{~nm}$ in water. The unit on the scale of colorbar represents the magnitude of field intensity in the absence of nanoparticle. The interior of the sphere was blackened.

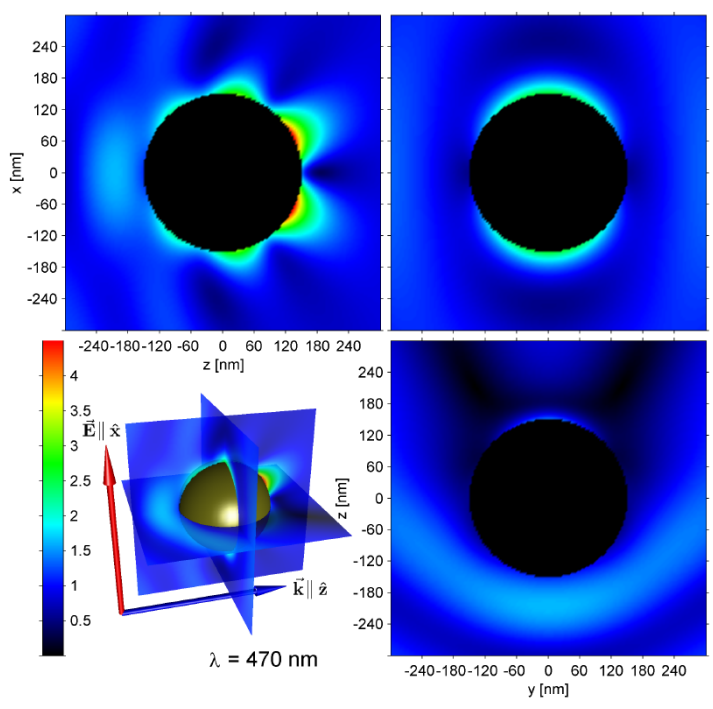

Fig. 3. Local electric field distribution around a single silver sphere with radius $a=150 \mathrm{~nm}$ in water.

A graphic illustration of such situation is shown in Fig. 4, where the results of calculations of electric field distribution around an ensemble of two silver spheres are presented.

The field enhancement at the mid-point inside $1 \mathrm{~nm}$ gap between two $39 \mathrm{~nm}$ silver nanoparticles aligned along the electric field direction can reach a few hundreds (see Fig. 5A). At even smaller distance between metallic nanoparticles the electric field enhancement can exceed three orders of magnitude.

According to the classical interpretation of SERS the enhancement of signal is proportional to the square of the local field magnitude at the frequency of incident

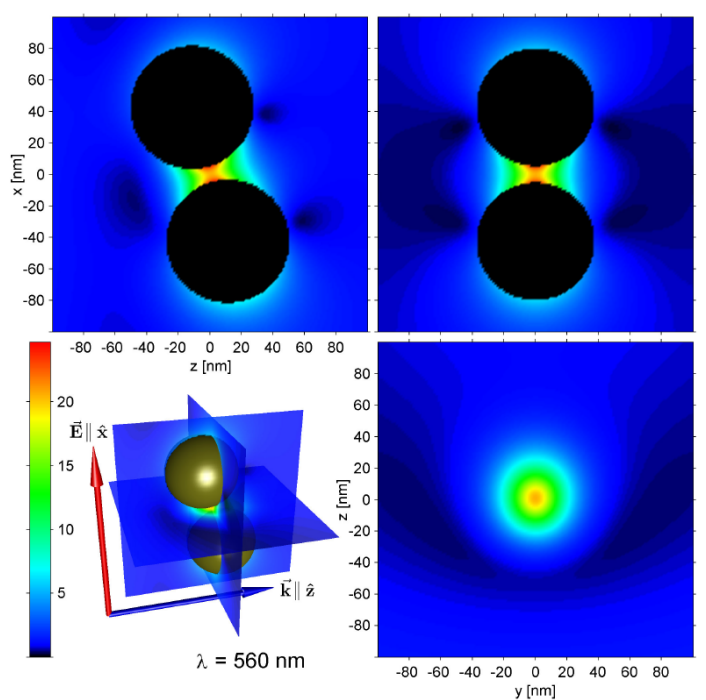

Fig. 4. Local electric field distribution around an ensemble of two spherical silver nanoparticles with radius $a=39 \mathrm{~nm}$ placed in water. The distance between nanospheres is $10 \mathrm{~nm}$.
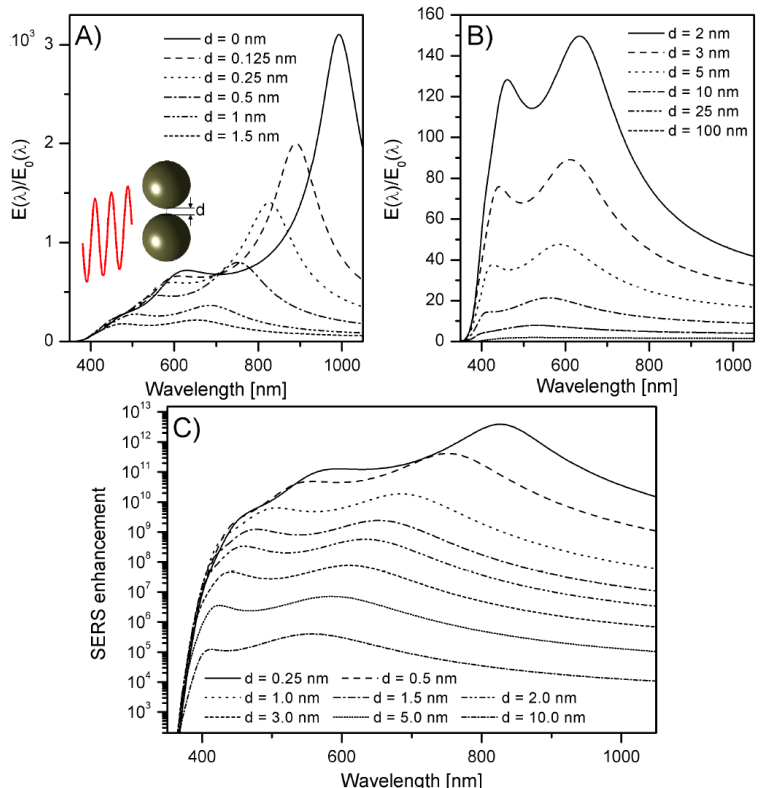

Fig. 5. Enhancement of electric field at the middle point between two $39 \mathrm{~nm}$ silver spheres (A, B) and corresponding SERS enhancement (C) caused by EM mechanism. Symbol $d$ denotes the distance between nanospheres. Calculations were performed using GMM method.

$\left(\omega_{\mathrm{e}}\right)$ and scattered (Stokes-shifted; $\left.\omega_{\mathrm{s}}\right)$ radiation

$$
E F_{\mathrm{EM}}=\left|\frac{E_{\mathrm{loc}}\left(\omega_{\mathrm{e}}\right)}{E_{0}}\right|^{2}\left|\frac{E_{\mathrm{loc}}\left(\omega_{\mathrm{s}}\right)}{E_{0}}\right|^{2} .
$$

If the plasmon resonance band is broad and the difference between the incident and Stokes-shifted frequency is small, then the approximation $E F_{\mathrm{EM}} \propto\left|E_{\mathrm{loc}}\left(\omega_{\mathrm{e}}\right) / E_{0}\right|^{4}$ is valid. The intensity of light scattered in SERS process 
by molecules placed in "hot spot" can be enhanced up to $10^{11}-10^{12}$ times. The approximate dependence of the enhancement factor of SERS (due to EM mechanism) on the wavelength of incident light is presented in Fig. 5C.

\section{Extinction spectra of silver sol}

The prerequisite for the occurrence of SERS phenomenon is electromagnetic excitations of surface plasmons in nanostructures forming the SERS active system. Very useful criterion for assessment and predicting the potential SERS-activity of silver colloid is a visual inspection of its extinction spectrum. It should have a broad band, whose source is the absorption and scattering of light by surface plasmons. The position and shape of this band depends on the kind of metal, from which nanostructures are built, and on the size and shape of these nanostructures. A colloidal dispersion containing non-aggregated silver nanoparticles exhibits maximal extinction in the violet and blue range. The extinction band broadens and shifts toward longer wavelengths with the increase of the size of particles. A similar dependence is observed for colloidal gold. The only difference is that the isolated gold nanoparticles most strongly absorb and scatter green and yellow light.
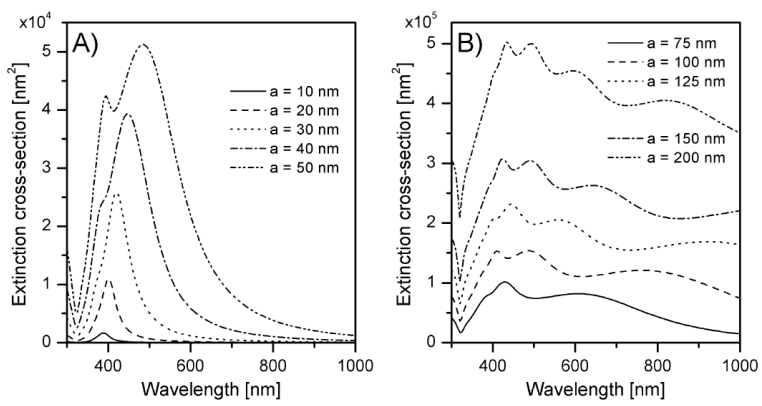

Fig. 6. Extinction spectra of water suspension of silver nanoparticles of various radius $a$ calculated on the basis of the Mie theory.

Extinction spectra can be calculated on the basis of the Mie theory (see Fig. 6). According to this theory the extinction cross-sections of homogeneous diamagnetic sphere of radius $a$ and dielectric permittivity $\varepsilon$ placed in the medium of permittivity $\varepsilon_{\text {med }}$ can be expressed as a sum of series of multipolar terms

$$
C_{\text {ext }}(\lambda, a)=\frac{2 \pi}{k^{2}} \sum_{n=1}^{\infty}(2 n+1) \operatorname{Re}\left(a_{n}+b_{n}\right),
$$

where $k=(2 \pi / \lambda) \sqrt{\varepsilon_{\text {med }}(\lambda)}$. $\lambda$ is the wavelength in vacuum. The coefficients $a_{n}$ and $b_{n}$ of this infinite series are obtained from appropriate boundary conditions at the surface of the sphere and are given by

$$
a_{n}=\frac{m \psi_{n}(m x) \psi_{n}^{\prime}(x)-\psi_{n}(x) \psi_{n}^{\prime}(m x)}{m \psi_{n}(m x) \xi_{n}^{\prime}(x)-\xi_{n}(x) \psi_{n}^{\prime}(m x)}
$$

$$
b_{n}=\frac{\psi_{n}(m x) \psi_{n}^{\prime}(x)-m \psi_{n}(x) \psi_{n}^{\prime}(m x)}{\psi_{n}(m x) \xi_{n}^{\prime}(x)-m \xi_{n}(x) \psi_{n}^{\prime}(m x)},
$$

where $x=k r$ and $m=\sqrt{\varepsilon(\omega) / \varepsilon_{\text {med }}(\omega)}$ are the size parameter and relative refractive index, respectively. $\psi_{n}$ and $\xi_{n}$ are the Riccatti-Bessel functions defined as $\psi_{n}(z)=z j_{n}(z)$ and $\xi_{n}(z)=z\left[j_{n}(z)+\mathrm{i} y_{n}(z)\right]$, where $j_{n}$ and $y_{n}$ are spherical Bessel functions of the first and second kind.

\section{Experimental}

\subsection{Materials}

Silver nitrate $\left(\mathrm{AgNO}_{3}\right)$, trisodium citrate $\left(\mathrm{Na}_{3} \mathrm{C}_{6} \mathrm{H}_{5} \mathrm{O}_{7} \cdot 2 \mathrm{H}_{2} \mathrm{O}\right)$, potassium chloride $(\mathrm{KCl})$ and hydrochloric acid $(\mathrm{HCl})$ were obtained from $\mathrm{POCh}$ S.A. (Poland). Rhodamine 6G and rhodamine B were purchased from Sigma-Aldrich. Milli-Q grade water $(18.2 \mathrm{M} \Omega \mathrm{cm})$ was used for the preparation of all solutions.

\subsection{Silver colloids production and samples preparation}

The nanoparticles used in experiments were prepared by chemical reduction of silver nitrate with trisodium citrate using a modified Lee and Meisel method [20]. Mechanism of reaction can be rewritten as follows:

$$
\begin{aligned}
& 4 \mathrm{Ag}^{+}+\mathrm{Na}_{3} \mathrm{C}_{6} \mathrm{H}_{5} \mathrm{O}_{7}+2 \mathrm{H}_{2} \mathrm{O} \\
& \quad \rightarrow 4 \mathrm{Ag}^{0}+\mathrm{H}_{3} \mathrm{C}_{6} \mathrm{H}_{5} \mathrm{O}_{7}+3 \mathrm{Na}^{+}+\mathrm{H}^{+}+\mathrm{O}_{2} \uparrow .
\end{aligned}
$$

The Lee and Meisel method allows to obtain the nanoparticles with desired sizes depending on the temperature at which suspension was kept during preparation of the colloid. For studies carried out in this work silver nanoparticles are obtained by mixing of $50 \mathrm{ml}$ of $1 \mathrm{mM}$ silver nitride with $1 \mathrm{ml}$ of $1 \%$ aqueous solution of trisodium citrate. The last solution was added in 4 portions. First portion $(0.1 \mathrm{ml})$ was added after silver nitride solution heating to temperature of $90^{\circ} \mathrm{C}$. Next solution was heated to about $96^{\circ} \mathrm{C}$ and after $20 \mathrm{~min}$, the second portion equal to $0.3 \mathrm{ml}$ was added. The next portions (3th and 4 th) each equal to $0.3 \mathrm{ml}$ were added after next 15 and $30 \mathrm{~min}$, respectively. After adding the last portion, the temperature was still kept at $96^{\circ} \mathrm{C}$ for about $30 \mathrm{~min}$, and content of flask was occasionally stirred. Finally, the resultant mixture was cooled down to room temperature and pure water was added to compensate the losses due to evaporation. Obtained mixtures were milky gray-green color and remained stable for a long time (up to several months). For further studies (extinction, particle sizing by dynamically scattered light (DLS) and SERS) they were 6 -fold diluted in water.

$\mathrm{KCl}$ or $\mathrm{HCl}$, respectively, were used as aggregating agents. All samples containing colloids with appropriate amount of one of these compounds were shaken on vortex stirrer for $30 \mathrm{~s}$ after addition. Then the analyte (rhodamine $6 \mathrm{G}$ or rhodamine $\mathrm{B}$ ) was added and mixing was continued for next $30 \mathrm{~s}$. The measurements were started immediately after the preparation of each sample. 


\subsection{Instrumentation}

The absorbance spectra were collected using a two-beam V550 (Jasco Inc., USA) spectrophotometer in standard $10 \mathrm{~mm}$ path length optical glass cells.

The particle sizes were determined by photon correlation spectroscopy. Fluctuations of the intensity of DLS were analysed on the system composed of a photon counting unit Photocor-PC1 (based on Hamamatsu R6358-10 photomultiplier) and 288-channels Photocor-FC correlator working in multiple-tau spacing mode (both devices with dedicated software were purchased from Photocor Instruments Inc., USA). The power of the incident beam (632.8 nm light from a He-Ne laser source) was adjusted with circular variable neutral density filter (Thorlabs Inc., USA) and a width of the beam was reduced by using lenses and pinholes. Scattering angle was set to $90^{\circ}$. The laser light was polarized perpendicularly to the scattering plane. The scattered light was accumulated for $15 \mathrm{~s}$. Obtained autocorrelation functions were analyzed using DynaLS software (Alango Ltd., Israel).

The Raman spectra were recorded on DK480 (Spectral Products Inc., USA), $0.5 \mathrm{~m}$ spectrometer equipped with ST6 CCD camera (Santa Barbara Instrument Group Inc., USA). Both devices were controlled by KestrelSpec software (Catalina Scientific Corp., USA). The spectra were excited by $632.8 \mathrm{~nm}$ line of $35 \mathrm{~mW} \mathrm{He}-\mathrm{Ne}$ laser (Coherent Inc., USA) at $30 \mathrm{~s}$ exposure.

\section{Results and discussion}

\subsection{SERS on aggregated silver nanoparticles}

The Raman scattering of molecules in solutions containing non-aggregated silver nanoparticles exhibits relatively weak enhancement. The average intensity of the Raman scattering from molecules of analytes (both rhodamines) dissolved in colloidal suspension grows up to 2 orders of magnitude. This enhancement factor is insufficient for practical SERS applications so partial aggregation of colloid is necessary [21].

The increase in chloride concentration leads to a significant reduction of the main extinction band related to the dipole plasmon resonance of unaggregated nanoparticles (as one can see in Fig. 7A), while a new absorption band arises beyond $600 \mathrm{~nm}$ and then increases and shifts towards longer wavelengths. The appearance of the second band proves that the silver aggregates (initially dimers and then aggregates of larger size) are formed. This problem is in more detail discussed in authors' previous paper [22].

As one can see in Fig. 7B the results of consecutive DLS measurements are strongly correlated with the changes in absorption spectra. For $\mathrm{KCl}$ concentration ranging from $3.33 \mathrm{mM}$ to $33.3 \mathrm{mM}$ the measured average radius of aggregates increased from slightly less than $50 \mathrm{~nm}$ to about $70 \mathrm{~nm}$. These relatively small changes in modal value of the presented particle size distributions suggest that during the first minute after preparation of the samples
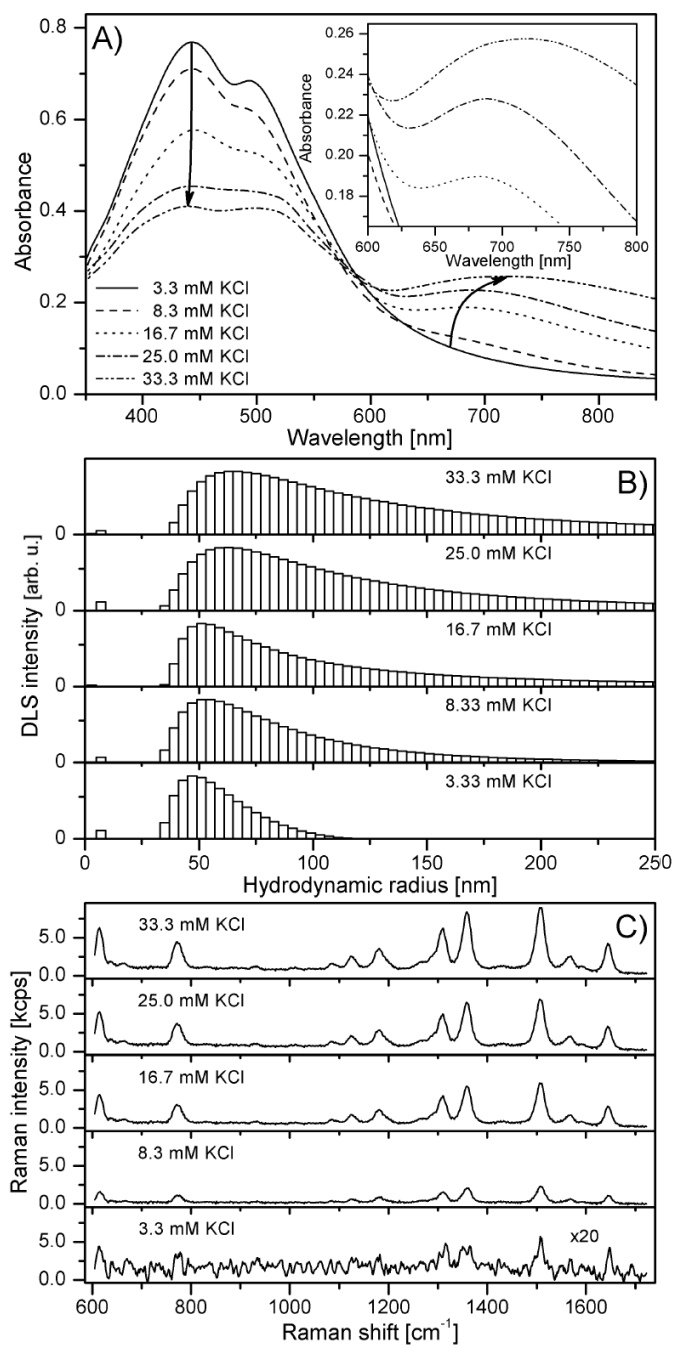

Fig. 7. Extinction spectra (A) of silver colloids containing $0.8 \mu \mathrm{M}$ rhodamine $6 \mathrm{G}$ with addition of various amounts of $\mathrm{KCl}$. Corresponding results of DLS measurements (B) and SERS spectra (C).

most of the nanoparticles still remain unaggregated or form only small clusters. It is also noteworthy that the coexistence of $\mathrm{KCl}$ and rhodamine $6 \mathrm{G}$ (at the concentration of the order of $\mu \mathrm{M}$ ) in colloid leads to the slightly greater extent of aggregation than in the case of silver sol with $\mathrm{KCl}$ but without dye.

The presence in silver sol both rhodamine $6 \mathrm{G}$ and small amount of chloride results in the appearing of new absorption band of the dye at about $500 \mathrm{~nm}$, while free rhodamine 6G exhibits the peak of absorption at about $527 \mathrm{~nm}$. The changes in the absorption spectrum and quenching of the fluorescence emission (the results are presented in [23]) indicate that cationic molecules of rhodamine $6 \mathrm{G}$ in colloidal $\mathrm{Ag} /$ chloride ions mixture tend to concentrate close to metallic nanoparticles and easily adsorb on their negatively charged surfaces at preferred orientation with respect to them [24]. It can facilitate the dimerization of dye molecules [25]. 
$1.7 \mathrm{mM}$ concentration of chloride is still not sufficient to effective SERS activation of silver sol and, in consequence, no Raman scattering of $0.8 \mu \mathrm{M}$ Rh6G was recorded. As the content of chloride increases SERS intensity of rhodamine $6 \mathrm{G}$ on silver nanoparticles also strongly increases and reaches the maximum at $33.3 \mathrm{mM}$ $\mathrm{KCl}$ concentration (see Fig. $7 \mathrm{C}$ ). At this concentration of chloride ions the system seems to undergo a metastable regime and the longer laser irradiation (just tens of seconds) leads to the noticeable degradation of the colloid. It seriously limits the time available for recording Raman spectra. Further enlargement of the amount of chloride leads to the instability and gradual precipitation of colloid. It also results in the considerable decrease of SERS intensity.

\subsection{Time dependence of SERS signal}

Figure 8 shows the temporal evolution of absorbance spectra obtained from samples of $100 \mathrm{nM}$ rhodamine $\mathrm{B}$ in silver sol. One can see that during at least two hours observations the extinction of $\mathrm{Ag}$ colloid $/ \mathrm{HCl} /$ rhodamine $\mathrm{B}$ solution decreases across almost the whole measured spectrum, while the plasmon resonance band slightly shifts towards higher energy. This shift is probably related to the changes of charge density on the surface of nanoparticles as a result of adsorption of chloride ions. Figure 8 also shows the evolution of spectra in the red and near infrared region where the second wide absorption band arises. These changes are mostly expressed for the $\mathrm{HCl}$ concentration of $10 \mathrm{mM}$. As in the case of Ag nanoparticles $/ \mathrm{KCl} /$ rhodamine $6 \mathrm{G}$ systems this second band indicates the appearance in solution of small assemblies of closely spaced nanoparticles. It is clearly seen that the red band in extinction spectrum shifts to longer wavelengths with time. This shift is related to the increasing size of aggregates. Excluding the sample with the lower content of $\mathrm{HCl}$, after a certain time the colloids exhibit nearly equal extinction in almost the whole spectral range indicating a large extent of aggregation.

For all concentrations of $\mathrm{HCl}$ the changes in extinction spectrum (especially in the red region) are correlated with the alteration of the Raman intensity (see Fig. 9B). The increase of the concentration of $\mathrm{HCl}$ results in earlier achievement of the maximum of the Raman intensity.

In the case of rhodamine $\mathrm{B}$ on the citrate reduced silver sol a replacement of $\mathrm{KCl}$ by $\mathrm{HCl}$ as an aggregating agent results in considerable increase in SERS intensity (over 10 -fold growth in comparison to $\mathrm{KCl}$ ). The possible explanation of this spectacular effect is a transition of the dye molecules from zwitterionic to cationic form caused by the decrease of $\mathrm{pH}[26]$. It is not observed for rhodamine $6 \mathrm{G}$.

DLS measurements (Fig. 9C and D) clearly show that the aggregation proceeds faster for higher concentration of aggregating agent. The fairly rapid decline in the intensity of DLS at higher chloride concentration is probably the result of the reduction in the number of scattering objects due to sedimentation of large aggregates.

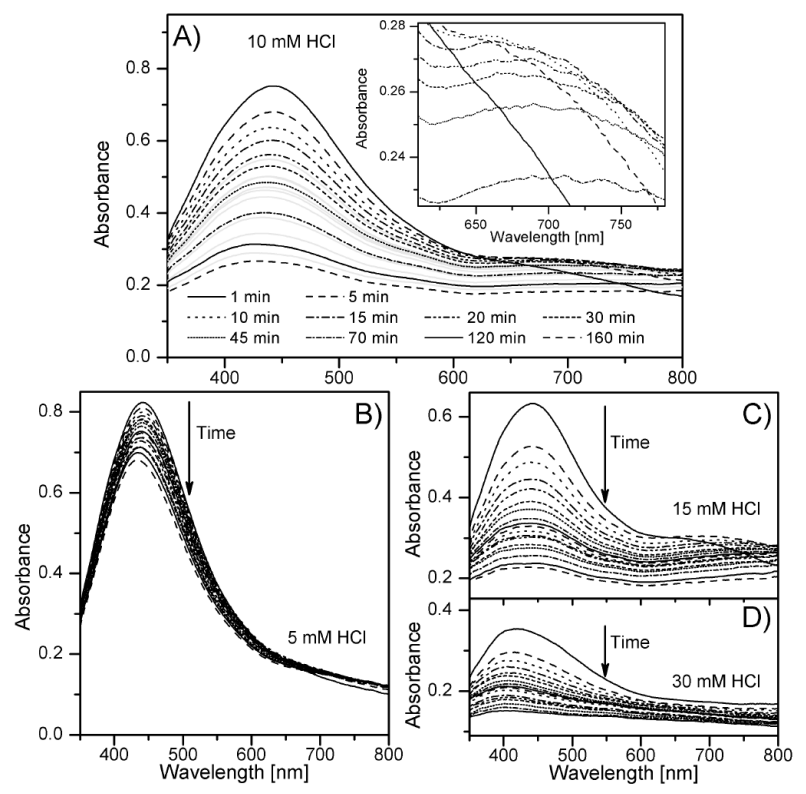

Fig. 8. Sets of extinction spectra of the citrate reduced $\mathrm{Ag}$ colloid with $0.1 \mu \mathrm{M}$ rhodamine $\mathrm{B}$ and various concentrations of $\mathrm{HCl}$.
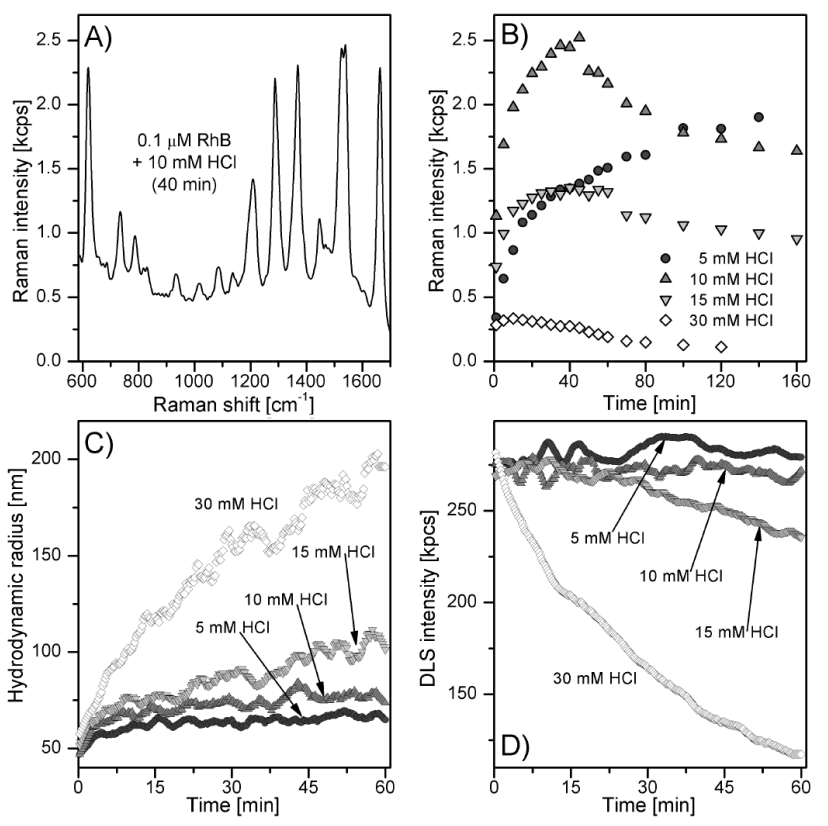

Fig. 9. SERS spectra of $0.1 \mu \mathrm{M}$ rhodamine $\mathrm{B}$ on silver sol with added $10 \mathrm{mM} \mathrm{HCl}$ at $40 \mathrm{~min}$ after preparation of the sample (A), the time-dependent changes of the intensity of the most pronounced Raman peak of rhodamine $\mathrm{B}$ (at about $1530 \mathrm{~cm}^{-1}$ ) recorded for the samples containing various amount of $\mathrm{HCl}(\mathrm{B})$. The evolution of the mean hydrodynamic radius of aggregates (C) and the intensity of dynamically scattered light (D) during first hour of the observation. 
The strong correlation between presented DLS results and changes of the intensity of the Raman signal confirms that the aggregation of nanoparticles is necessary to achieve an effective SERS enhancement.

\subsection{SERS enhancement factor}

Both rhodamine $6 \mathrm{G}$ and rhodamine $\mathrm{B}$ are strongly fluorescent compounds so due to intense background of fluorescence emission it is hard to precisely estimate an enhancement factor of the Raman signal. The intensity of vibrational bands is very weak in comparison to fluorescence intensity and it causes the main difficulty. The enhancement factor can be described as

$$
E F=\frac{I_{\mathrm{SERS}}}{C_{\mathrm{SERS}}} \frac{C_{\mathrm{NR}}}{I_{\mathrm{NR}}},
$$

where $C_{\mathrm{SERS}}$ and $C_{\mathrm{NR}}$ are the concentrations of analyte for SERS and normal Raman measurement, respectively, and $I_{\text {SERS }}$ and $I_{\mathrm{NR}}$ are the corresponding intensity of certain vibration. In presented work the maximal enhancement factor roughly estimated with the above formula was about $10^{7}$ in the case of rhodamine $6 \mathrm{G}$ and colloid aggregated by $\mathrm{KCl}$. Almost $10^{8}$-fold increase of the Raman signal was observed only for rhodamine B adsorbed on silver colloid treated with moderate amount of $\mathrm{HCl}$. The smallest amount of rhodamine B for which the distinct SERS spectra was recorded was $1 \mathrm{nM}$.

The observed SERS enhancement is, in fact, an average over a very broad distribution of enhancements present in all places occupied by molecules of rhodamines within the whole scattering volume. As one can conclude from Fig. 5C the enhancement factor can range from values near unity to values exceeding $10^{11}$.

\section{Conclusions}

The theoretical predictions as well as the experimental results presented in this work show a decisive role of the aggregation of nanoparticles in its SERS-activity. The prerequisite for the aggregation is a significant lowering of the energy barrier to the attraction between particles. In the case of negatively charged Lee-Meisel sol chloride are able to promote this process. Colloidal solution with the addition of small amount of chloride is characterized by relatively good stability and controllability of the aggregation. However, due to the various kinetics of this process under different ionic strength of solutions it is hard to determine the optimal moment for SERS measurement.

Chloride ions not only effectively affect EM enhancement of SERS but also contribute to CT mechanism. Due to the co-adsorption with rhodamines studied here chlorides allow the dye molecules to interact directly with silver surface, that results in the additional enhancement of the Raman scattering.

\section{References}

[1] M. Fleishman, P.J. Hendra, A.J. McQuillan, Chem. Phys. Lett. 26, 163 (1974).

[2] D.L. Janmaire, R.P. Van Duyne, Electroanal. Chem. 84, 1 (1977).

[3] M.G. Albrecht, J.A. Creighton, J. Am. Chem. Soc. 84, 5215 (1977).

[4] J.A. Creighton, C.G. Blatchford, M.G. Albrecht, J. Chem. Soc. Faraday Trans. 75, 790 (1979).

[5] X. Huang, P.K. Jain, J.H. El-Sayed, M.A. El-Sayed, Nanomedicine 2, 681 (2007).

[6] W. Cai, T. Gao, H. Hong, J. Sun, Nanotechnol., Sci. Appl. I, 17 (2008).

[7] S. Lee, S. Kim, J. Choo, S.Y. Shin, Y.H. Lee, H.Y. Choi, S. Ha, K. Kang, C.H. Oh, Anal. Chem. 79, 916 (2007).

[8] G. Maltzahn, J. Park, A. Agrawal, N.K. Bandaru, S.K. Das, M.J. Sailor, S.N. Bhatia, Cancer Res. 69, 3892 (2009).

[9] S. Kruszewski, Surf. Interface Anal. 21, 830 (1994).

[10] S. Kruszewski, Cryst. Res. Technol. 41, 562 (2006).

[11] A. Derkachova, K. Kolwas, Eur. J. Phys. Special Topics 144, 93 (2007).

[12] A.L. Gonzalez, C. Noguez, Phys. Status Solidi C 4, 4118 (2007)

[13] H. Ditlbacher, N. Felidj, J.R. Krenn, B. Lamprecht, A. Leitner, F.R. Aussenegg, Appl. Phys. B 73, 373 (2001).

[14] K. Kneipp, Y. Wang, H. Kneipp, L.T. Perelman, I. Itzkan, R.R. Dasari, M.S. Feld, Phys. Rev. Lett. 78, 1667 (1997).

[15] S. Nie, S.R. Emory, Science 275, 1102 (1997).

[16] G. Mie, Ann. Phys. 25, 377 (1908).

[17] Y.-L. Xu, Appl. Opt. 34, 4573 (1995).

[18] Y.-L. Xu, B.Å.S. Gustafson, Recent Res. Devel. Optics 3, 599 (2003).

[19] Z.B. Wang, B.S. Luk'yanchuk, M.H. Hong, Y. Lin, T.C. Chong, Phys. Rev. B 70, 035418 (2004).

[20] P.C. Lee, D. Meisel, J. Phys. Chem. 86, 3391 (1982).

[21] H. Bengter, C. Tengroth, S.P. Jacobsson, J. Raman Spectrosc. 36, 1015 (2005).

[22] S. Kruszewski, M. Cyrankiewicz, Acta Phys. Pol. A 119, 114 (2011).

[23] M. Cyrankiewicz, S. Kruszewski, J. Phys., Conf. Ser. 289, 012016 (2011).

[24] M.A. Noginov, M. Vondrova, S.M. Williams, M. Bahoura, V.I. Gavrilenko, S.M. Black, V.P. Drachev, V.M. Shalaev, A. Sykes, J. Opt. A, Pure Appl. Opt. 7, 219 (2005).

[25] V.M. Martinez, F.L. Arbeloa, J.B. Prieto, I.L. Arbeloa, J. Phys. Chem. B 109, 7443 (2005).

[26] S. Woislawski, J. Am. Chem. Soc. 76, 5201 (1953). 